\title{
Segmentation under geometrical conditions using geodesic active contours and interpolation using level set methods
}

\author{
Christian Gout ${ }^{\mathrm{a}}$, Carole Le Guyader ${ }^{\mathrm{a}}$ and Luminita Vese ${ }^{\mathrm{b}}$ \\ ${ }^{a}$ INSA de Rouen, Laboratoire de Mathématiques de l'INSA, Place Emile Blondel, BP 08, \\ 76131 Mont-Saint-Aignan Cedex, France \\ E-mail: chris_gout@cal.berkeley.edu,carole.leguyader@laposte.net \\ ${ }^{b}$ University of California, Los Angeles, Department of Mathematics, 405, Hilgard Avenue, Los Angeles, \\ CA 90095-1555, USA \\ E-mail: lvese@math.ucla.edu
}

Received 19 September 2003; accepted 25 March 2004

\begin{abstract}
Let $I: \Omega \rightarrow \Re$ be a given bounded image function, where $\Omega$ is an open and bounded domain which belongs to $\Re^{n}$. Let us consider $n=2$ for the purpose of illustration. Also, let $S=\left\{x_{i}\right\}_{i} \in \Omega$ be a finite set of given points. We would like to find a contour $\Gamma \subset \Omega$, such that $\Gamma$ is an object boundary interpolating the points from $S$. We combine the ideas of the geodesic active contour (cf. Caselles et al. [7,8]) and of interpolation of points (cf. Zhao et al. [40]) in a level set approach developed by Osher and Sethian [33]. We present modelling of the proposed method, both theoretical results (viscosity solution) and numerical results are given.
\end{abstract}

Keywords: geodesic active contour, level set method, viscosity solution, interpolation of points

AMS subject classification: 49L25, 74G65, 68U10

\section{Introduction}

In this paper, we aim at combining the ideas developed in the geodesic active contour approach [8] with a geometrical approach including interpolation constraints. These interpolation conditions can be well data in geophysics (see [3,22]) or can be used to help the segmentation process when it is needed because of the image (see, for instance, [29]).

This work will be done in the context of the level set approach, developed by Osher and Sethian ([32-34] or in [1,9,11,12,25,26,30,31,36,37,39] and others), which consists in considering the problem in a higher dimension, and more precisely, considering the evolving curve as the zero level set of a function. When using this method, the topology changes, cusps, and corners are allowed. Moreover, we do not have to deal with the issue of parameterization anymore since we work on a fixed rectangular grid for the discretization. It is an intrinsic representation. The process exposed consists first in 


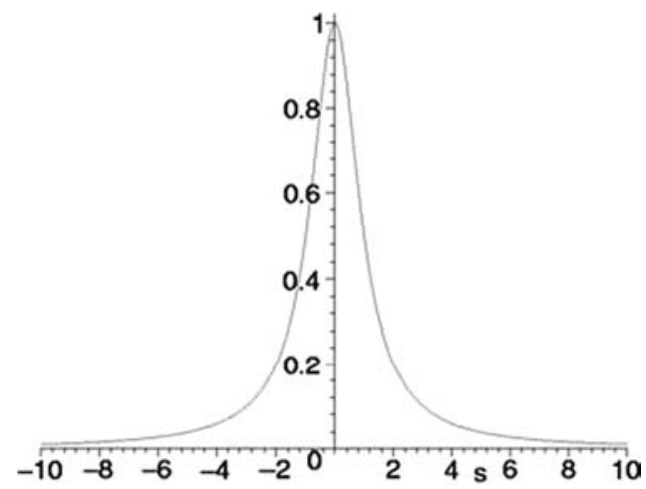

Figure 1. Representation of the function $g$.

minimizing an energy which contains a term in connection with the a priori knowledge of the image and another term linked to the geometrical constraints. Edges are assumed to be pixels where the gradient intensity function varies abruptly. The Euler-Lagrange theorem gives us the partial differential equation satisfied by the function. The equation can be seen as a mean-curvature-flow-like problem, except that it includes a function linked to the geometrical and image information.

\section{Statement of the problem}

\subsection{Model}

Let $I: \Omega \rightarrow \Re$ be a given bounded image function, with $\Omega$ an open bounded subset of $\Re^{n}$. Let us consider $n=2$ for the purpose of illustration. As mentioned in the introduction, we plan to introduce a geometrical approach in this new method by adding interpolation constraints. Thus let $S=\left(x_{i}, y_{i}\right)_{i} \in \Omega$ be a finite set of given points close to the boundary we want to determine. We would like to find a contour $\Gamma \subset \Omega$ such that $\Gamma$ is the boundary of the object under consideration, interpolating the points from $S$ which belong to this boundary. Let $g:[0,+\infty[\rightarrow[0,+\infty[$ be an edge-function as in $[6,11,31]$, such that $g(0)=1, g$ is positive, strictly decreasing and $\lim _{s \rightarrow \infty} g(s)=0$. The function $g$ is applied to the gradient of the image $|\nabla I(x, y)|$. An example of such a function is given by

$$
g(s)=\frac{1}{1+s^{2}},
$$

so

$$
g(|\nabla I(x, y)|)=\frac{1}{1+|\nabla I(x, y)|^{2}} .
$$


Furthermore, to the set of points $S$, we associate the distance function $d(a)$ from every point $a \in \Omega$ to $S$

$$
d(a)=\operatorname{distance}(a, S)=\min _{c \in S}|a-c| .
$$

By definition, $d(a)=0$ if and only if $a \in S$. In order to find a contour $\Gamma$ such that $g \simeq 0$ or $d \simeq 0$ on $\Gamma$, we propose to minimize the following energy:

$$
E(\Gamma)=\int_{\Gamma} d \cdot g(|\nabla I|) \mathrm{d} s
$$

We will start with an initial guess $\Gamma_{0}$ and we will apply gradient descent to the energy, in a level set approach. We will construct a family of curves $\Gamma(t)$ decreasing the energy as $t$ increases.

\section{Method}

\subsection{The level set approach}

The level set approach [32-34] consists in considering the evolving active contour $\Gamma=\Gamma(t)$ as the zero level set of a function $\Phi$, which is a Lipschitz continuous function defined by:

$$
\left\{\Phi: \begin{array}{l}
\Omega \times[0,+\infty[\longrightarrow \Re \\
(x, y, t) \longmapsto \Phi(x, y, t)
\end{array}\right.
$$

such that

$$
\Gamma(t)=\{(x, y) \in \Omega: \Phi(x, y, t)=0\},
$$

and $\Phi(\cdot, \cdot, t)$ takes opposite signs on each side of $\Gamma(t)$. It enables us to re-write the energy in terms of $\Phi$ as follows

$$
F(\Phi)=\int_{\Omega} d(x, y) g(|\nabla I(x, y)|)|\nabla H(\Phi(x, y))| \mathrm{d} x \mathrm{~d} y,
$$

where $H$ is the one-dimensional Heaviside function. By approximating $H$ by a $C^{1}$ or $C^{2}$ regularization $H_{\varepsilon}$, as $\varepsilon \rightarrow 0$ and letting $\delta_{\varepsilon}=H_{\varepsilon}^{\prime}$, the energy can be written as (see [12])

$$
F_{\varepsilon}(\Phi)=\int_{\Omega} d(x, y) g(|\nabla I(x, y)|) \delta_{\varepsilon}(\Phi)|\nabla \Phi(x, y)| \mathrm{d} x \mathrm{~d} y,
$$

where

$$
\int_{\Omega} \delta_{\varepsilon}(\Phi)|\nabla \Phi(x, y)| \mathrm{d} x \mathrm{~d} y
$$

is the length of the zero level set of $\Phi$. 


\subsection{Minimization of the energy}

In this section, we minimize the energy $F_{\varepsilon}$ and we determine the associated partial differential equation satisfied by $\Phi$. To this end, we use variational calculus with the extension of the classical Euler-Lagrange theorem to a function that depends on two variables. Then, we propose a proof based on the Gâteaux derivative.

\subsubsection{Using Euler-Lagrange theorem}

Theorem 1 (Euler-Lagrange (Theorem 37.1.2 de Dubrovin et al. [18])). Let $D$ denote a region with piecewise smooth boundary $\partial D$, of the Euclidean space $\Re^{n}$ with Euclidean coordinates $x^{1}, \ldots, x^{n}$. Consider the linear space $F$ of smooth vector-functions $f\left(x^{1}, \ldots, x^{n}\right)=\left(f^{1}, \ldots, f^{k}\right)$ defined on $D$. Let $L\left(x^{\beta} ; p^{j} ; q_{\alpha}^{i}\right)$ be a smooth real-valued function of the three arguments $x^{\beta}, 1 \leqslant \beta \leqslant n, p^{j}, 1 \leqslant j \leqslant k, q_{\alpha}^{i}, 1 \leqslant i \leqslant k$, $1 \leqslant \alpha \leqslant n$. We construct a functional $I[f]$ defined on $F$ as follows:

$$
I[f]=\int_{D} L\left(x^{\beta} ; f^{j}\left(x^{\beta}\right) ; f_{x^{\alpha}}^{i}\left(x^{\beta}\right)\right) \mathrm{d} x^{1} \wedge \mathrm{d} x^{2} \wedge \cdots \wedge \mathrm{d} x^{n} .
$$

A function $f_{0} \in F$ is extremal for the functional $I[f]$ if and only if it satisfies the system of equations

$$
\frac{\delta I[f]}{\delta f^{i}}=\frac{\partial L}{\partial f_{0}^{i}}-\sum_{\alpha=1}^{n} \frac{\partial}{\partial x^{\alpha}}\left(\frac{\partial L}{\partial f_{0, x^{\alpha}}^{i}}\right), \quad i \in\{1, \ldots, k\} .
$$

In the considered model, we have

$$
L\left(x, y, \Phi, \frac{\partial \Phi}{\partial x}, \frac{\partial \Phi}{\partial y}\right)=\delta_{\varepsilon}(\Phi) d(x, y) g(|\nabla I(x, y)|)|\nabla \Phi(x, y)| .
$$

It follows

$$
\left\{\begin{array}{l}
\frac{\partial L}{\partial \Phi}=\delta_{\varepsilon}^{\prime}(\Phi) d(x, y) g(|\nabla I(x, y)|)|\nabla \Phi|, \\
\frac{\partial L}{\partial p}=\delta_{\varepsilon}(\Phi) d(x, y) g(|\nabla I(x, y)|) \frac{\partial \Phi / \partial x}{|\nabla \Phi|} \\
\frac{\partial L}{\partial q}=\delta_{\varepsilon}(\Phi) d(x, y) g(|\nabla I(x, y)|) \frac{\partial \Phi / \partial y}{|\nabla \Phi|}
\end{array}\right.
$$

with $p=\partial \Phi / \partial x$ and $q=\partial \Phi / \partial y$.

Thus we get

$\frac{\partial L}{\partial \Phi}-\frac{\partial}{\partial x}\left[\frac{\partial L}{\partial p}\right]-\frac{\partial}{\partial y}\left[\frac{\partial L}{\partial q}\right]=0 \quad \Longleftrightarrow \quad \delta_{\varepsilon}(\Phi) \operatorname{div}\left(d(x, y) g(|\nabla I(x, y)|) \frac{\nabla \Phi}{|\nabla \Phi|}\right)=0$.

The related evolution equation is thus given by

$$
\frac{\partial \Phi}{\partial t}=\delta_{\varepsilon}(\Phi) \operatorname{div}\left(\mathbf{d}(\mathbf{x}, \mathbf{y}) \mathbf{g}(|\nabla \mathbf{I}(\mathbf{x}, \mathbf{y})|) \frac{\nabla \Phi}{|\nabla \Phi|}\right)
$$


We now propose an alternative proof (using Gâteaux derivative) of the evolution equation (6) which will additionally give boundary conditions.

\subsubsection{Gâteaux derivative}

We recall that $F$ is differentiable in the Gateaux sense at $\Phi \in X$ if the application

$$
x \longmapsto F_{\Phi}^{\prime}(\Psi)=\lim _{h \rightarrow 0} \frac{F(\Phi+h \Psi)-F(\Phi)}{h}
$$

is defined for any $\Psi \in X$ and if it is linear and continuous. In this case, Riesz theorem provides the existence of $F^{\prime}(\Phi) \in X$ such that $F_{\Phi}^{\prime}(\Psi)=\left\langle F^{\prime}(\Phi), \Psi\right\rangle, F^{\prime}(\Phi)$ being the gradient of $F$ at $\Phi$.

Coming back to the problem, let us determine the Gâteaux derivative of the energy $F_{\varepsilon}$. The Gâteaux derivative of $F_{\varepsilon}$ with respect to $\Phi$ in the direction of $\Psi$ is

$$
F_{\varepsilon_{\Phi}}^{\prime}(\Psi)=\lim _{h \rightarrow 0} \frac{F_{\varepsilon}(\Phi+h \Psi)-F_{\varepsilon}(\Phi)}{h},
$$

where

$$
\begin{aligned}
F_{\varepsilon}(\Phi+h \Psi)-F_{\varepsilon}(\Phi)= & \int_{\Omega} d(x, y) g(|\nabla I(x, y)|) \delta_{\varepsilon}(\Phi+h \Psi)|\nabla \Phi+h \nabla \Psi| \mathrm{d} x \mathrm{~d} y \\
& -\int_{\Omega} d(x, y) g(|\nabla I(x, y)|) \delta_{\varepsilon}(\Phi)|\nabla \Phi| \mathrm{d} x \mathrm{~d} y .
\end{aligned}
$$

Then

$$
\begin{aligned}
F_{\varepsilon}( & +h \Psi)-F_{\varepsilon}(\Phi) \\
= & \int_{\Omega} d(x, y) g(|\nabla I(x, y)|) \delta_{\varepsilon}(\Phi+h \Psi)|\nabla \Phi| \sqrt{1+h^{2} \frac{|\nabla \Psi|^{2}}{|\nabla \Phi|^{2}}+2 h \frac{\langle\nabla \Phi, \nabla \Psi\rangle}{|\nabla \Phi|^{2}}} \mathrm{~d} x \mathrm{~d} y \\
& -\int_{\Omega} d(x, y) g(|\nabla I(x, y)|) \delta_{\varepsilon}(\Phi)|\nabla \Phi| \mathrm{d} x \mathrm{~d} y
\end{aligned}
$$

and we use a Taylor development (on $h$ ) to linearize the square root. Taking the limit when $h \rightarrow 0$, we get

$$
\begin{aligned}
F_{\varepsilon_{\Phi}}^{\prime}(\Psi)= & \int_{\Omega} d(x, y) g(|\nabla I(x, y)|) \delta_{\varepsilon}^{\prime}(\Phi) \Psi|\nabla \Phi| \mathrm{d} x \mathrm{~d} y \\
& +\int_{\Omega} d(x, y) g(|\nabla I(x, y)|) \delta_{\varepsilon}(\Phi) \frac{\langle\nabla \Phi, \nabla \Psi\rangle}{|\nabla \Phi|} \mathrm{d} x \mathrm{~d} y .
\end{aligned}
$$

Hence

$$
\begin{aligned}
F_{\varepsilon_{\Phi}}^{\prime}(\Psi)= & -\int_{\Omega} \delta_{\varepsilon}(\Phi) \operatorname{div}\left(d(x, y) g(|\nabla I(x, y)|) \frac{\nabla \Phi}{|\nabla \Phi|}\right) \Psi \mathrm{d} x \mathrm{~d} y \\
& +\int_{\partial \Omega} d(x, y) g(|\nabla I(x, y)|) \frac{\delta_{\varepsilon}(\Phi)}{|\nabla \Phi|} \Psi\left(\frac{\partial \Phi}{\partial x} v_{x} \mathrm{~d} \sigma+\frac{\partial \Phi}{\partial y} v_{y} \mathrm{~d} \sigma\right) \mathrm{d} \sigma
\end{aligned}
$$


and

$$
\begin{aligned}
F_{\varepsilon_{\Phi}}^{\prime}(\Psi)= & -\int_{\Omega} \delta_{\varepsilon}(\Phi) \operatorname{div}\left(d(x, y) g(|\nabla I(x, y)|) \frac{\nabla \Phi}{|\nabla \Phi|}\right) \Psi \mathrm{d} x \mathrm{~d} y \\
& +\int_{\partial \Omega} d(x, y) g(|\nabla I(x, y)|) \frac{\delta_{\varepsilon}(\Phi)}{|\nabla \Phi|} \frac{\partial \Phi}{\partial v} \Psi \mathrm{d} \sigma .
\end{aligned}
$$

This expression must vanish for all $\Psi$ in order to satisfy the Euler-Lagrange equation. Therefore, we obtain the following problem:

$$
\left\{\begin{array}{l}
\delta_{\varepsilon}(\Phi) \operatorname{div}\left(d(x, y) g(|\nabla I(x, y)|) \frac{\nabla \Phi}{|\nabla \Phi|}\right)=0 \\
\text { with the boundary conditions } \frac{\delta_{\varepsilon}(\Phi)}{|\nabla \Phi|} \frac{\partial \Phi}{\partial v}=0 .
\end{array}\right.
$$

The evolution problem is thus the one given by (6).

As stressed by Zhao et al. [40], there is a balance between the potential force and the surface tension. A parallel can be drawn with the classical deformable models, a model which shows off an equilibrium between the regularization energy and the energy linked to the image. The closer we are to the finite set of points or on edges, the more important is the flexibility in the model, since in this case the expression $d(x, y) g(|\nabla I(x, y)|)$ vanishes.

Proposition 2. The energy $\mathrm{F}_{\varepsilon}(\Phi)$ is decreasing with time $t$.

Proof. We follow the same arguments as Zhao et al. [41].

We see in (6) that, when a local minimum is reached, then the quantity $\partial \Phi / \partial t$ tends to 0 , which means that the model converges. Indeed, the steady state is reached and the curve no longer evolves. A constant $\alpha$ can be added to the equation (6) to increase the speed of convergence, which is obtained from an additional area constraint as done in [12], and a rescaling can be made so that the motion is applied to all level sets by replacing $\delta_{\varepsilon}$ by $|\nabla \Phi|$. As stressed by Zhao et al. [40] and Alvarez et al. [2], it makes the flow independent of the scaling of $\Phi$. Thus the proposed model is, for any $(x, y) \in \Omega$

$$
\left\{\begin{array}{l}
\Phi(x, y, 0)=\Phi_{0}(x, y), \\
\frac{\partial \Phi}{\partial t}=|\nabla \Phi|\left[\operatorname{div}\left(d(x, y) g(|\nabla I(x, y)|) \frac{\nabla \Phi}{|\nabla \Phi|}\right)-\alpha\right] .
\end{array}\right.
$$

This model is then an active contour model based on the mean curvature flow motion, to which we have included the interpolation constraints through the distance function. The evolution equation can be formally re-written as

$$
\begin{aligned}
\frac{\partial \Phi}{\partial t}= & |\nabla \Phi| d(x, y) g(|\nabla I(x, y)|) \operatorname{div}\left(\frac{\nabla \Phi}{|\nabla \Phi|}\right) \\
& +\langle\nabla(d(x, y) g(|\nabla I(x, y)|)), \nabla \Phi\rangle-\alpha|\nabla \Phi| .
\end{aligned}
$$


The parabolic problem with the associated boundary conditions $\partial \Phi / \partial \nu=0$ on $\partial \Omega$ (Neumann Condition) with $v$ denoting the unit exterior normal to the boundary of $\Omega$ is thus defined by

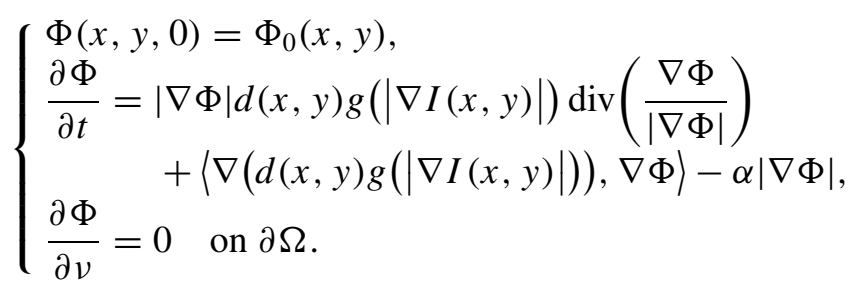

The term $\kappa=\operatorname{div}(\nabla \Phi /|\nabla \Phi|)$ being the level set representation of the mean curvature, equation (8) can be rewritten by

$$
\left\{\begin{array}{l}
\Phi(x, y, 0)=\Phi_{0}(x, y), \\
\frac{\partial \Phi}{\partial t}=|\nabla \Phi| d(x, y)(|\nabla I(x, y)|) \kappa+\langle\nabla(d(x, y) g(|\nabla I(x, y)|)), \nabla \Phi|-\alpha| \nabla \Phi|, \\
\frac{\partial \Phi}{\partial v}=0 \quad \text { on } \partial \Omega .
\end{array}\right.
$$

Some remarks can be formulated as regards the partial differential equation satisfied by $\Phi$.

1. For this remark, we refer the reader to the works of Caselles et al. [8]. For an ideal edge, we have $|\nabla I| \cong \infty, g=0$ and the curve stops, which is in practice unrealistic. Here, we have refined the criterion so that the curve stops with the term

$$
\langle\nabla(d(x, y) g(|\nabla I(x, y)|)), \nabla \Phi\rangle
$$

which naturally appears in the model and that uses jointly the gradient of the edgefunction coupled with the distance function. This is more accurate than the mere gradient in particular if the variations of the gradient along a boundary are different (cf. [8]). Besides, it takes both, information linked with the image (whether we are or not on a boundary) and information linked with the geometrical constraints (whether we are near or not the set of given points).

2. We have

$$
\frac{\partial \Phi}{\partial t}=|\nabla \Phi|\left[\operatorname{div}\left(d(x, y) g(|\nabla I(x, y)|) \frac{\nabla \Phi}{|\nabla \Phi|}\right)-\alpha\right] .
$$

All the level sets move according to

$$
\Gamma_{t}=d(x, y) g(|\nabla I(x, y)|) \kappa \vec{n}-\langle\nabla(d(x, y) g(|\nabla I(x, y)|)), \vec{n}\rangle \vec{n}-\alpha \vec{n},
$$

with $\kappa=\operatorname{div}(\nabla \Phi /|\nabla \Phi|)$ and $\vec{n}=-(\nabla \Phi /|\nabla \Phi|)$ (interior normal). Indeed, we have for all level sets, $\Phi(\Gamma(t), t)=$ constant.

Calculating the derivatives with respect to the variable $t$ yields

$$
\frac{\mathrm{d}}{\mathrm{d} t}[\Phi(\Gamma(t), t)]=0 \quad \Longleftrightarrow \Phi_{t}+\left\langle\nabla \Phi, \Gamma_{t}\right\rangle=0 .
$$


where $\Phi_{t}$ denotes $\partial \Phi / \partial t$ and $\Gamma_{t}, \partial \Gamma / \partial t$. Hence, using the definition of $\Gamma_{t}$ and $\vec{n}$, we get

$$
\Phi_{t}=d(x, y) g(|\nabla I(x, y)|) \kappa|\nabla \Phi|+\langle\nabla(d(x, y) g(|\nabla I(x, y)|)), \nabla \Phi\rangle-\alpha|\nabla \Phi| .
$$

3. In the right-hand side of equation (8), $\nabla(d(x, y) g(|\nabla I(x, y)|))$ is well-defined, except at the points that are equidistant from at least two points of the given finite set $S$. Indeed, the function $d(a)=d(a, S)$ is continuous as the inf of a finite number of continuous functions but at these equidistant points the solution is nondifferentiable and the gradient is not defined.

The distance function $d$ satisfies the Eikonal equation $|\nabla d|=1$. In the theoretical part devoted to the existence and uniqueness of the solution to the considered problem, we need a certain smoothness on the distance function $d$. Using the curvature as a regularizing or smoothing term enables us to get the desired properties on $d$. What follows is taken from Sethian's book [34]: the main conclusion that we use here is that "a front propagating at the speed $1-\varepsilon \kappa$ for $\varepsilon>0$ does not form corners and remains smooth for all time. Furthermore, as the dependence on curvature vanishes, the limit of this motion is the entropy-satisfying solution obtained for the constant speed case".

In the next section, we aim at proving the existence and uniqueness of the solution to this parabolic problem, with the theory of viscosity.

\subsection{Existence, uniqueness of the solution to the problem - Viscosity theory}

\subsubsection{General background}

In this section, we recall the notion of viscosity solutions. The general background is widely taken from the 'User's guide to viscosity solutions' (by Crandall et al. [17]). This theory applies to some partial differential equations that can formally be written in the form $F\left(x, u, D u, D^{2} u\right)$ where $D u$ denotes the gradient and $D^{2} u$ the Hessian matrix (which is symmetric). In general, $F$ is defined as

$$
F: \Re^{n} \times \Re \times \Re^{n} \times S(n) \longrightarrow \Re,
$$

where $S(n)$ denotes the set of symmetric $(n \times n)$ matrices.

In our case, $F$ will be defined by $F: \Omega \times \Re \times \Re^{2} \times S(2) \rightarrow \Re$. Two conditions (monotonicity conditions) are necessary to apply this theory to an equation of the type $F=0$, namely:

1. $F(x, r, p, X) \leqslant F(x, s, p, X)$ with $r \leqslant s$.

2. $F(x, r, p, Y) \leqslant F(x, r, p, X)$ with $Y \geqslant X$.

The second condition is called degenerate ellipticity. When both conditions hold, $F$ is said to be proper. Coming back to the problem, we plan to check if these conditions are satisfied by the model. We note that for parabolic problems, if $(x, r, p, X) \rightarrow$ 
$F(t, x, r, p, X)$ is proper for a fixed $t \in[0, T]$, then so is the associated parabolic problem:

$$
u_{t}+F\left(t, x, u, D u, D^{2} u\right)=0
$$

In our case, we have

$$
\begin{aligned}
\Phi_{t} & -|\nabla \Phi| d(x, y) g(|\nabla I(x, y)|) \operatorname{div}\left(\frac{\nabla \Phi}{|\nabla \Phi|}\right) \\
& -\langle\nabla(d(x, y) g(|\nabla I(x, y)|)), \nabla \Phi\rangle+\alpha|\nabla \Phi|=0 .
\end{aligned}
$$

Carrying out the differentiations yields

$$
\begin{aligned}
\Phi_{t} & -|\nabla \Phi| d(x, y) g(|\nabla I(x, y)|) \\
& \times\left(\frac{\frac{\partial^{2} \Phi}{\partial x^{2}}+\frac{\partial^{2} \Phi}{\partial y^{2}}}{|\nabla \Phi|}-\frac{\left(2 \frac{\partial \Phi}{\partial x} \frac{\partial \Phi}{\partial y} \frac{\partial^{2} \Phi}{\partial x \partial y}+\left(\frac{\partial \Phi}{\partial y}\right)^{2} \frac{\partial^{2} \Phi}{\partial y^{2}}+\left(\frac{\partial \Phi}{\partial x}\right)^{2} \frac{\partial^{2} \Phi}{\partial x^{2}}\right)|\nabla \Phi|^{-1}}{|\nabla \Phi|^{2}}\right) \\
& -\langle\nabla(d(x, y) g(|\nabla I(x, y)|)), \nabla \Phi\rangle+\alpha|\nabla \Phi|=0 .
\end{aligned}
$$

For $p=\left(\begin{array}{c}p_{1} \\ p_{2}\end{array}\right) \in \mathfrak{R}^{2}$, we denote by $(p \otimes p) /|p|^{2}$ the matrix defined by

$$
\frac{p \otimes p}{|p|^{2}}=\frac{1}{|p|^{2}}\left(\begin{array}{cc}
p_{1}^{2} & p_{1} p_{2} \\
p_{1} p_{2} & p_{2}^{2}
\end{array}\right)
$$

We have

$$
\begin{aligned}
F(x, y, r, p, X)= & -d(x, y) g(|\nabla I(x, y)|) \operatorname{trace}(X) \\
& +d(x, y) g(|\nabla I(x, y)|) \operatorname{trace}\left(\frac{p \otimes p}{|p|^{2}} X\right) \\
& -\langle\nabla(d(x, y) g(|\nabla I(x, y)|)), p\rangle+\alpha|p| .
\end{aligned}
$$

We then get

$$
\begin{aligned}
F(x, y, r, p, X)= & -d(x, y) g(|\nabla I(x, y)|) \operatorname{trace}\left(\left(I-\frac{p \otimes p}{|p|^{2}}\right) X\right) \\
& -\langle\nabla(d(x, y) g(|\nabla I(x, y)|)), p\rangle+\alpha|p| .
\end{aligned}
$$

It is obvious that the first condition is satisfied since it does not depend explicitly on $r$, so $F(x, y, r, p, X)=F(x, y, p, X)$. Let us analyze the degenerate ellipticity. We have then to compare $F(x, y, p, X)$ and $F(x, y, p, Y)$ when $Y \geqslant X$, that is to compare

$$
-d(x, y) g(|\nabla I(x, y)|) \operatorname{trace}\left(\left(I d-\frac{p \otimes p}{|p|^{2}}\right) X\right)
$$

and

$$
-d(x, y) g(|\nabla I(x, y)|) \operatorname{trace}\left(\left(I d-\frac{p \otimes p}{|p|^{2}}\right) Y\right),
$$


when the inequality holds for $X$ and $Y$. The set of symmetric matrices is equipped with the usual order, which means that

$$
X \leqslant Y \quad \Longleftrightarrow \quad \forall \xi \in \mathfrak{R}^{2}-\left\{0_{\mathfrak{R}^{2}}\right\}, \quad \xi^{\mathrm{T}} X \xi \leqslant \xi^{\mathrm{T}} Y \xi .
$$

Let us denote by $A(p)$ the matrix defined by

$$
A(p)=I d-\frac{p \otimes p}{|p|^{2}}
$$

which corresponds to

$$
A=\left(\begin{array}{cc}
\frac{p_{2}^{2}}{|p|^{2}} & -\frac{p_{1} p_{2}}{|p|^{2}} \\
-\frac{p_{1} p_{2}}{|p|^{2}} & \frac{p_{1}^{2}}{|p|^{2}}
\end{array}\right) .
$$

We suppose as well that $p \neq 0$ since we have a singularity for $p=0$. The marix $A(p)$ is positive because

$$
\begin{aligned}
& \forall \xi \in \mathfrak{R}^{2}-\left\{0_{\mathfrak{R}^{2}}\right\} \quad \text { such that } \xi=\left(\begin{array}{l}
\xi_{1} \\
\xi_{2}
\end{array}\right): \\
& \xi^{\mathrm{T}} A(p) \xi=\frac{1}{|p|^{2}}\left(\xi_{1} p_{2}-\xi_{2} p_{1}\right)^{2} \geqslant 0 .
\end{aligned}
$$

The matrix $A(p)$ is symmetric positive. Its eigenvalues are positive and there exists an orthonormal basis such that $D=P^{\mathrm{T}} A P$, with $P$ an orthogonal matrix and $D$ a diagonal matrix with positive values. Thus one can write $A=\sigma \sigma^{\mathrm{T}}$, with $\sigma$ the matrix defined by $\sigma=P D^{1 / 2}$. Coming back to our problem, we have, with the following notations:

$$
\operatorname{trace}(A X)=\operatorname{trace}\left(\sigma \sigma^{\mathrm{T}} X\right)=\operatorname{trace}\left(\sigma^{\mathrm{T}} X \sigma\right)
$$

and

$$
\operatorname{trace}(A X)=\sum_{i=1}^{2} \sigma_{i}^{\mathrm{T}} X \sigma_{i}, \quad \text { with } \sigma_{i} \text { the } i \text { th column of } \sigma .
$$

Suppose that $Y \geqslant X$. We can easily deduce that

$$
\forall i \in\{1,2\}, \quad \sigma_{i}^{\mathrm{T}} X \sigma_{i} \leqslant \sigma_{i}^{\mathrm{T}} Y \sigma_{i} .
$$

The functions $d$ and $g$ being positive, we conclude that:

$$
-d(x, y) g(|\nabla I(x, y)|) \operatorname{trace}(A(p) X) \geqslant-d(x, y) g(|\nabla I(x, y)|) \operatorname{trace}(A(p) Y) .
$$

We have then proved that for $p \neq 0, F$ is degenerate elliptic.

This is the general framework in which the viscosity theory has been first introduced. We then suppose that for all theorems stated below, $F$ is proper and continuous. 
In [17] and [5], the notion of viscosity is introduced, with an example using the maximum principle, as follows: $F$ is proper, as already said. Suppose that $u \in C^{2}(O)$ and that

$$
F\left(x, u(x), D u(x), D^{2} u(x)\right) \leqslant 0 \quad \forall x \in \Re^{n} .
$$

Suppose that $\varphi$ is also $C^{2}(O)$ ( $O$ being an open subset of $\Re^{n}$ ) and $\hat{x} \in O$ is a local maximum of $u-\varphi$. This implies that $D(u-\varphi)(\hat{x})=0$ and $D^{2}(u-\varphi)(\hat{x}) \leqslant 0$, so

$$
\left\{\begin{array}{l}
D u(\hat{x})=D \varphi(\hat{x}), \\
D^{2} u(\hat{x}) \leqslant D^{2} \varphi(\hat{x}) .
\end{array}\right.
$$

Using the property of degenerate ellipticity of $F$, we get

$$
F\left(\hat{x}, u(\hat{x}), D \varphi(\hat{x}), D^{2} \varphi(\hat{x})\right) \leqslant F\left(\hat{x}, u(\hat{x}), D u(\hat{x}), D^{2} u(\hat{x})\right) \leqslant 0 .
$$

We can now give a definition of viscosity solutions:

Definition 1. Let $u \in C(O)$, then it is a viscosity solution to $F=0$ if and only if:

$$
\begin{aligned}
& \forall \Phi \in C^{2}(O), \quad \text { if } x_{0} \text { is a local maximum of } u-\Phi, \text { we have the relation } \\
& F\left(x_{0}, u\left(x_{0}\right), D \Phi\left(x_{0}\right), D^{2} \Phi\left(x_{0}\right)\right) \leqslant 0
\end{aligned}
$$

and

$$
\begin{aligned}
& \forall \Phi \in C^{2}(O), \quad \text { if } x_{0} \text { is a local minimum of } u-\Phi, \text { we have the relation } \\
& F\left(x_{0}, u\left(x_{0}\right), D \Phi\left(x_{0}\right), D^{2} \Phi\left(x_{0}\right)\right) \geqslant 0 .
\end{aligned}
$$

If $u$ only satisfies the first (second) inequality, then $u$ is said to be a viscosity subsolution (viscosity supersolution).

Crandall et al. [17] and Barles [5] give another definition based on the notions of superjet and subjet. In view of the above, we have for $x$ near $\hat{x}$,

$$
u(x) \leqslant u(\hat{x})-\varphi(\hat{x})+\varphi(x)
$$

and Taylor expansion gives ( $\varphi$ being $C^{2}$ )

$$
u(x) \leqslant u(\hat{x})+\langle p, x-\hat{x}\rangle+\frac{1}{2}\langle X(x-\hat{x}), x-\hat{x}\rangle+\mathrm{o}\left(|x-\hat{x}|^{2}\right), \quad x \longrightarrow \hat{x},
$$

where $p=D \varphi(\hat{x})$ and $X=D^{2} \varphi(\hat{x})$. We say that if $u: O \rightarrow \Re, \hat{x} \in O$ and (11) is satisfied as $O \ni x \rightarrow \hat{x},(p, X) \in J_{O}^{2,+} u(\hat{x}), J_{O}^{2,+} u(\hat{x})$ being the second order superjet of $u$ at $\hat{x}$.

Definition 2. A viscosity subsolution to $F=0$ on $O$ is a function $u \in U S C(O)$ such that:

$$
F(x, u(x), p, X) \leqslant 0 \quad \forall x \in O,(p, X) \in J_{O}^{2,+} u(x) .
$$


A viscosity supersolution to $F=0$ on $O$ is a function $v \in L S C(O)$ such that:

$$
F(x, v(x), p, X) \geqslant 0 \quad \forall x \in O,(p, X) \in J_{O}^{2,-} v(x) .
$$

We say that $u$ is a viscosity solution if it is both a subsolution and a supersolution. $\operatorname{USC}(O)$ is the set of upper semicontinuous functions on $O$ and $\operatorname{LSC}(O)$ is the set of lower semicontinuous functions on $O$.

Consider the following parabolic problem:

$$
u_{t}+F\left(t, x, u, D u, D^{2} u\right)=0 .
$$

For parabolic problems, Crandall et al. [17] extend these definitions. Here we assume that $D u$ and $D^{2} u$ stand for $D_{x} u(t, x)$ and $D_{x}^{2} u(t, x)$. Instead of working on $O$, we work on $\left.O_{T}=\right] 0, T[\times O$.

In [17], the parabolic variants of $J_{O}^{2,+}$ and $J_{O}^{2,-}$ are denoted by $P_{O}^{2,+}, P_{O}^{2,-}$, respectively. Thus $P_{O}^{2,+} u$ is defined as follows: $(a, p, X) \in \Re \times \Re^{n} \times S(n)$ lies in $P_{O}^{2,+} u(s, z)$ if $(s, z) \in O_{T}$ and

$$
\begin{aligned}
u(t, x) \leqslant & u(s, z)+a(t-s)+\langle p, x-z\rangle+\frac{1}{2}\langle X(x-z), x-z\rangle \\
& +\mathrm{o}\left(|t-s|+|x-z|^{2}\right) \quad \text { as } O_{T} \ni(t, x) \longrightarrow(s, z) .
\end{aligned}
$$

We will use the following definition of [17].

Definition 3. A subsolution to the parabolic equation on $O_{T}$ is a function $u \in U S C\left(O_{T}\right)$ such that

$$
a+F(t, x, u(t, x), p, X) \leqslant 0 \quad \text { for }(t, x) \in O_{T} \text { and }(a, p, X) \in P_{O}^{2,+} u(t, x) .
$$

A supersolution to the parabolic equation on $O_{T}$ is a function $v \in L S C\left(O_{T}\right)$ such that

$$
a+F(t, x, v(t, x), p, X) \geqslant 0 \quad \text { for }(t, x) \in O_{T} \text { and }(a, p, X) \in P_{O}^{2,-} v(t, x) .
$$

3.3.2. Existence and uniqueness of the solution to the problem: proof based on Ishii and Sato's work

Here is a first result that will be used in the sequel.

Preliminary. Let $p, q \in \Re^{n} \backslash\{0\}$, then

$$
\left|\frac{p}{|p|}-\frac{q}{|q|}\right| \leqslant \frac{|p-q|}{\min (|p|,|q|)}
$$

Proof. Suppose that $\min (|p|,|q|)=|p|$. Hence, the preliminary result is equivalent to

$$
\left|p-\frac{|p|}{|q|} q\right|^{2} \leqslant|p-q|^{2}
$$


We propose to prove that

$$
2\left(1-\frac{|p|}{|q|}\right)(p, q) \leqslant|q|^{2}-|p|^{2}
$$

Recall that $(1-|p| /|q|) \in[0,1]$, so using Cauchy-Schwartz inequality, we get

$$
2\left(1-\frac{|p|}{|q|}\right)(p, q) \leqslant 2|p|(|q|-|p|) \text {. }
$$

We deduce that

$$
2\left(1-\frac{|p|}{|q|}\right)(p, q) \leqslant|q|^{2}-|p|^{2},
$$

since $|p| \leqslant|q|$ and so $2|p|(|q|-|p|) \leqslant|q|^{2}-|p|^{2}$. The proposition is then proved.

We use here the existence theorem for viscosity solutions introduced by Ishii and Sato in [23]. This article treats the difficult case of singular parabolic equations with non-linear oblique derivative boundary conditions while we wish to apply it to a problem with homogeneous Neumann boundary conditions. As formerly done in their article, we denote by

$$
\rho(p, q)=\min \left(\frac{|p-q|}{\min (|p|,|q|)}, 1\right) .
$$

We assume that $\Omega$ is a bounded domain in $\Re^{n}$ with a $C^{1}$ boundary. Let us consider the following conditions:

1. $F \in C\left([0, T] \times \bar{\Omega} \times \Re \times\left(\Re^{n}-\{0\}\right) \times S^{n}\right)$, where $S^{n}$ denotes the space of $n \times n$ symmetric matrices equipped with the usual ordering.

2. There exists a constant $\gamma \in \Re$ such that for each $(t, x, p, X) \in[0, T] \times \bar{\Omega} \times$ $\left(\Re^{n}-\{0\}\right) \times S^{n}$, the function $u \mapsto F(t, x, u, p, X)-\gamma u$ is non decreasing on $\Re$.

3. For each $R>0$, there exists a continuous function $w_{R}:[0, \infty[\rightarrow[0, \infty[$ satisfying $w_{R}(0)=0$ such that if $X, Y \in S^{n}$ and $\mu_{1}, \mu_{2} \in[0, \infty[$ satisfy:

$$
\left(\begin{array}{cc}
X & 0 \\
0 & Y
\end{array}\right) \leqslant \mu_{1}\left(\begin{array}{cc}
I & -I \\
-I & I
\end{array}\right)+\mu_{2}\left(\begin{array}{cc}
I & 0 \\
0 & I
\end{array}\right)
$$

then

$$
\begin{aligned}
& F(t, x, u, p, X)-F(t, y, u, q,-Y) \\
& \quad \geqslant-w_{R}\left(\mu_{1}\left(|x-y|^{2}+\rho(p, q)^{2}\right)+\mu_{2}+|p-q|+|x-y|(1+\max (|p|,|q|))\right),
\end{aligned}
$$

for all $t \in[0, T], x, y \in \bar{\Omega}, u \in \Re$ with $|u| \leqslant R$ and $p, q \in \Re^{n} \backslash\{0\}$.

4. $B \in C\left(\Re^{n} \times \Re^{n}\right) \cap C^{1,1}\left(\Re^{n} \times\left(\Re^{n} \backslash\{0\}\right)\right)$.

5. For each $x \in \mathfrak{R}^{n}$, the function $p \mapsto B(x, p)$ is positively homogeneous of degree one in $p$, i.e., $B(x, \lambda p)=\lambda B(x, p), \forall \lambda \geqslant 0, p \in \Re^{n} \backslash\{0\}$. 
6. There exists a positive constant $\theta$ such that $\left\langle v(z), D_{p} B(z, p)\right\rangle \geqslant \theta$ for all $z \in \partial \Omega$ and $p \in \Re^{n}-\{0\}$. Here $v(z)$ denotes the unit outer normal vector of $\Omega$ at $z \in \partial \Omega$.

We recall the following theorem taken from [23].

Theorem 3. Consider the following problem:

$$
\begin{cases}u_{t}+F\left(t, x, u, D u, D^{2} u\right)=0 & \text { in }] 0, T[\times \Omega, \\ B(x, D u)=0 & \text { in }] 0, T[\times \partial \Omega\end{cases}
$$

satisfying $u(0, x)=g(x)$ for $x \in \bar{\Omega}$. Assume that conditions 1-6 hold. Then for each $g \in C(\bar{\Omega})$ there is a unique viscosity solution $u \in C([0, T[\times \bar{\Omega})$ of (13) satisfying $u(0, x)=g(x)$ for $x \in \bar{\Omega}$.

We apply this theorem to the considered problem. $F$ is defined by

$$
\begin{aligned}
F(t, x, u, p, X)= & -\operatorname{trace}\left(d(x) g(|\nabla I(x)|)\left(I-\frac{p \otimes p}{|p|^{2}}\right) X\right) \\
& -\langle\nabla(d(x) g(|\nabla I(x)|)), p\rangle .
\end{aligned}
$$

Denoting by $A(x, p)$ the symmetric positive matrix defined by

$$
A(x, p)=d(x) g(|\nabla I(x)|)\left(I-\frac{p \otimes p}{|p|^{2}}\right)
$$

we get

$$
F(t, x, u, p, X)=-\operatorname{trace}(A(x, p) X)-\langle\nabla(d(x) g(|\nabla I(x)|)), p\rangle .
$$

$F$ presents a singularity for $p=0$ but is continuous otherwise. The first point is satisfied. $F$ does not depend explicitly on $u$ so any negative constant $\gamma$ satisfies the second condition.

For the third point, the inequality (12) gives us that for all $r, s \in \mathfrak{R}^{2}$,

$$
(X r, r)+(Y s, s) \leqslant \mu_{1}|r-s|^{2}+\mu_{2}\left(|r|^{2}+|s|^{2}\right) .
$$

Taking successively $r=\sigma(x, p) e_{i}$ and $s=\sigma(y, q) e_{i}$ with $\left(e_{i}\right)_{i}$ an orthonormal basis of $\Re^{2}$ (as done in [21], $A(x, p)=\sigma(x, p) \sigma^{\mathrm{T}}(x, p)$ ), we get

$$
\begin{aligned}
& \operatorname{trace}(A(x, p) X)+\operatorname{trace}(A(y, q) Y) \\
& \leqslant \mu_{1} \operatorname{trace}\left((\sigma(x, p)-\sigma(y, q))(\sigma(x, p)-\sigma(y, q))^{\mathrm{T}}\right) \\
& \quad+\mu_{2}(d(x) g(|\nabla I(x)|)+d(y) g(|\nabla I(y)|)) .
\end{aligned}
$$

Hence

$$
\begin{aligned}
& \operatorname{trace}(A(x, p) X)+\operatorname{trace}(A(y, q) Y) \\
& \quad \leqslant \mu_{1}\left|\sqrt{d(x) g(|\nabla I(x)|)} \frac{p}{|p|}-\sqrt{d(y) g(|\nabla I(y)|)} \frac{q}{|q|}\right|^{2}+2 \theta \mu_{2} .
\end{aligned}
$$


The function $x \mapsto d(x) g(|\nabla I(x)|)$ is bounded by $\theta$.

Moreover, one has:

$$
\begin{aligned}
& \left|\sqrt{d(x) g(|\nabla I(x)|)} \frac{p}{|p|}-\sqrt{d(y) g(|\nabla I(y)|)} \frac{q}{|q|}\right|^{2} \\
& =\mid(\sqrt{d(x) g(|\nabla I(x)|)}-\sqrt{d(y) g(|\nabla I(y)|)}) \frac{p}{|p|} \\
& \quad+\left.\sqrt{d(y) g(|\nabla I(y)|)}\left(\frac{p}{|p|}-\frac{q}{|q|}\right)\right|^{2} .
\end{aligned}
$$

Thus

$$
\begin{aligned}
& \left|\sqrt{d(x) g(|\nabla I(x)|)} \frac{p}{|p|}-\sqrt{d(y) g(|\nabla I(y)|)} \frac{q}{|q|}\right|^{2} \\
& \quad \leqslant 2(\sqrt{d(x) g(|\nabla I(x)|)}-\sqrt{d(y) g(|\nabla I(y)|)})^{2}+2 d(y) g(|\nabla I(y)|)\left|\frac{p}{|p|}-\frac{q}{|q|}\right|^{2} .
\end{aligned}
$$

Using the preliminary and properties of the functions $x \mapsto d(x) g(|\nabla I(x)|)$ and $x \mapsto$ $\sqrt{d(x) g(|\nabla I(x)|)}$ as in [21], we can conclude that

$$
\operatorname{trace}(A(x, p) X)+\operatorname{trace}(A(y, q) Y) \leqslant \mu_{1}\left(2 \zeta|x-y|^{2}+8 \theta \rho(p, q)^{2}\right)+2 \theta \mu_{2} .
$$

Next, we have to evaluate the expression

$$
\begin{aligned}
& F(t, x, u, p, X)-F(t, y, u, q,-Y) \\
& =-(\operatorname{trace}(A(x, p) X)+\operatorname{trace}(A(y, q) Y)) \\
& \quad-(\langle\nabla(d(x) g(|\nabla I(x)|)), p\rangle-\langle\nabla(d(y) g(|\nabla I(y)|)), q\rangle) .
\end{aligned}
$$

Using the same arguments as in [21], we have

$$
\begin{aligned}
& |\langle\nabla(d(x) g(|\nabla I(x)|)), p\rangle-\langle\nabla(d(y) g(|\nabla I(y)|)), q\rangle| \\
& \quad \leqslant \kappa|x-y| \max (|p|,|q|)+C_{2}|p-q|,
\end{aligned}
$$

from which we deduce

$$
\begin{aligned}
& -(F(t, x, u, p, X)-F(t, y, u, q,-Y)) \\
& \quad \leqslant \mu_{1}\left[2 \zeta|x-y|^{2}+8 \theta \rho(p, q)^{2}\right]+2 \theta \mu_{2}+\kappa|x-y| \max (|p|,|q|)+C_{2}|p-q|
\end{aligned}
$$

and

$$
\begin{aligned}
& -(F(t, x, u, p, X)-F(t, y, u, q,-Y)) \\
& \leqslant \max \left(2 \zeta, 8 \theta, C_{2}, \kappa\right)\left(\mu_{1}\left(\rho(p, q)^{2}+|x-y|^{2}\right)\right. \\
& \left.\quad+\mu_{2}+|p-q|+|x-y|(1+\max (|p|,|q|))\right) .
\end{aligned}
$$

We just have to take $w_{R}(l)=\max \left(2 \zeta, 8 \theta, C_{2}, \kappa\right) l . w_{R}(0)=0$ and $w_{R}$ is nondecreasing on $[0, \infty[$. 


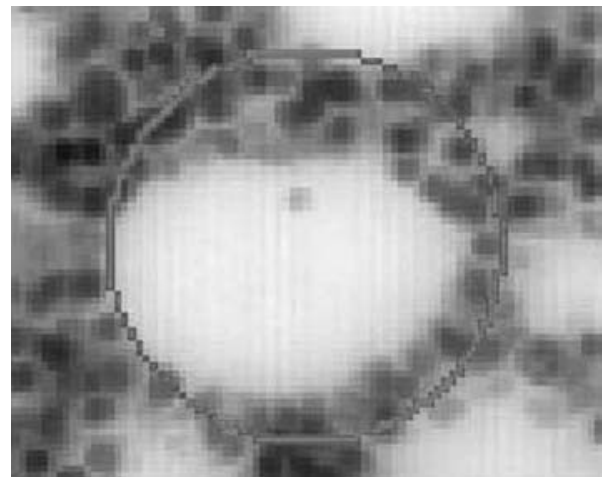

Figure 2. Initial condition.

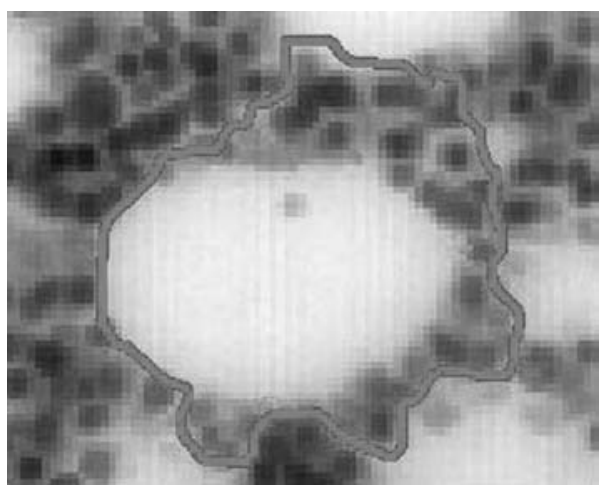

Figure 3. Using classical tool (snakes), we obtain the following result.

The fourth point is fulfilled with assumptions on $v$ (14).

Then, it is easy to check that $B$ is positively homogeneous of degree one. For the last point, one can easily see that

$$
B(z, p)=\langle v(z), p\rangle
$$

and

$$
\left\langle v(z),\left.D_{p} B(z, p)|=| v(z)\right|^{2}=1 .\right.
$$

We take $\theta=1$ and the last assumption is fulfilled.

\section{Numerical results}

In order to illustrate the proposed method, we give a numerical example. The interpolation conditions are useful in order to help the process when some image data are missing as shown in [29]. But the interpolation conditions also permit us to choose an initial condition which is far from the final result as shown in the following numerical example (image coming from Matlab). 


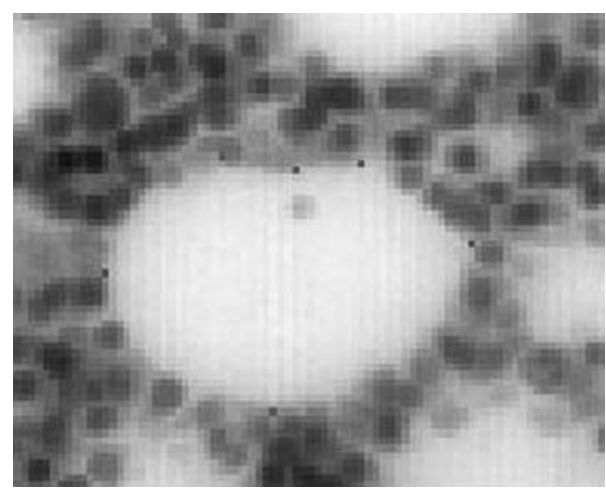

Figure 4. Interpolation conditions (6 points).

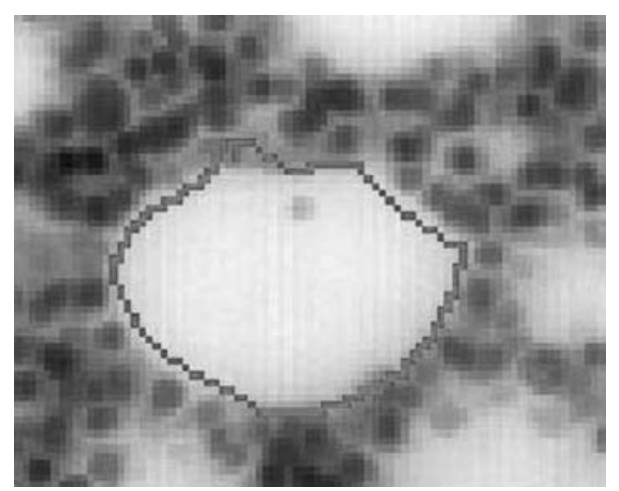

Figure 5. Final result using interpolation conditions of figure 4 and from the initial condition of figure 2.

In order to help the process, we give some interpolation conditions (6 points): We use the method proposed in this paper knowing that:

- The criterion that makes the algorithm stop can be either a preset number of iterations or a check that the solution is stationary.

- The distance is computed using the Fast Marching Method (see, for instance, [34]).

- The distance is normalized in order to have the same weight between a priori information of the image and geometrical constraints.

- The discretization is made using finite differences as done in [12].

- We have taken $\delta t=0.1$. The regularization term is equal to 0.8 . The number of iterations is 20 in this numerical example.

We obtain the result given in figure 5, which gives a good segmentation of the considered zone. Other numerical examples are given in [28]. 


\section{Acknowledgements}

The authors are very grateful to Guy Barles and Witold Respondek for valuable comments that helped to improve the manuscript.

Luminita Vese was supported in part by the National Science Foundation (ITR/AP Grant \#0113439), the National Institute of Mental Health and the National Institute of Neurological Disorders and Stroke (Grant \#MH65166), and by the Alfred P. Sloan Foundation. Christian Gout was supported in part by Dassault Sys (contract SLQ01192002).

\section{References}

[1] D. Adalsteinsson and J.A. Sethian, A fast level set method for propagating interfaces, J. Comput. Phys. 118(2) (1995) 269-277.

[2] L. Alvarez, P.-L. Lions and J.-M. Morel, Image selective smoothing and edge detection by nonlinear diffusion, SIAM J. Numer. Anal. 29(3) (1992) 845-866.

[3] D. Apprato, J.B. Betbeder, C. Gout and A. Vieira-Testé, Segmentation method under geometric constraints after pre-processing, in: Curves and Surfaces, Vol. IV, eds. A. Cohen, C. Rabut and L.L. Schumaker (Vanderbilt Univ. Press, Nashville, TN, 2000) pp. 9-18.

[4] G. Barles, Nonlinear Neumann Boundary Conditions for Quasilinear Degenerate Elliptic Equations and Applications, First version.

[5] G. Barles, Solutions de Viscosité des Équations de Hamilton-Jacobi (Springer, Berlin, 1994).

[6] V. Caselles, F. Catté, C. Coll and F. Dibos, A geometric model for active contours in image processing, Numer. Math. 66 (1993) 1-31.

[7] V. Caselles, R. Kimmel and G. Sapiro, Geodesic active contours, in: Proc. of the Fifth Internat. Conf. on Computer Vision (20-23 June 1995) pp. 694-699.

[8] V. Caselles, R. Kimmel and G. Sapiro, Geodesic active contours, Internat. J. Comput. Vision 22(1) (1997) 61-87.

[9] T.F. Chan, B.Y. Sandberg and L.A. Vese, Active contours without edges for vector-valued images, J. Visual Communication Image Representation 11(2) (2000) 130.

[10] T.F. Chan, J. Shen and L. Vese, Variational PDE models in image processing, Notices Amer. Math. Soc. 50(1) (2003) 14-26.

[11] T.F. Chan and L.A. Vese, An efficient variational multiphase motion for the Mumford-Shah segmentation mode, in: IEEE Asilomar Conf. on Signals Systems and Computers, Vol. 1 (2000) pp. 490-494.

[12] T. Chan and L. Vese, Active contours without edges, IEEE Trans. Image Process. 10(2) (2001) 266277.

[13] I. Cohen and L.D. Cohen, Deformable models for 3D medical images using finite element and balloons, in: Proc. of IEEE Conf. on Computer Vision and Pattern Recognition, Champaign (IEEE, Los Alamitos, 1992) pp. 592-598.

[14] I. Cohen, L.D. Cohen and N. Ayache, Using deformable surfaces to segment 3D images and infer differential structures, Computer Vision 56(2) (1992) 242-263.

[15] L.D. Cohen, On active contours models and balloons, Computer Vision 53(2) (1991) 211-218.

[16] L.D. Cohen, E. Bardinet and N. Ayache, Surface reconstruction using active contour models, INRIA, Rapport de Recherche (1992).

[17] M.G. Crandall, H. Ishii and P.-L. Lions, User's guide to viscosity solutions of second order partial differential equations, Bull. Amer. Math. Soc. 27(1) (1992) 1-69.

[18] B.A. Dubrovin, A.T. Fomenko and S.P. Novikov, Modern geometry methods and applications, Part I, in: The Geometry of Surfaces, Transformation Groups, and Fields (Springer, Berlin, 1992).

[19] A. Friedman, Partial Differential Equations of Parabolic Type (Prentice-Hall, Englewood Cliffs, NJ, 1964). 
[20] Y. Giga and M.-H. Sato, Generalized interface evolution with boundary condition, Proc. Japan Acad. Ser. A 67 (1991) 263-266.

[21] C. Gout, C. Le Guyader and L. Vese, Image segmentation under interpolation conditions, Preprint, CAM-IPAM, University of California at Los Angeles (2003) 44 pages.

[22] C. Gout and S. Vieira-Testé, An algorithm for contrast enhancement and segmentation of images, in: IEEE Internat. Conf. on Image Processing, Vol. 2, Vancouver, Canada (2001) pp. 716-719.

[23] H. Ishii and M.-H. Sato, Nonlinear oblique derivative problems for singular degenerate parabolic equations on a general domain, Preprint, Waseda (2001).

[24] M. Kass, A. Witkin and D. Terzopoulos, Snakes: Active contour models, Internat. J. Comput. Vision 1(4) (1987) 133-144.

[25] S. Kichenassamy, A. Kumar, P. Olver, A. Tannenbaum and A. Yezzi, Gradient flows and geometric active contour models, in: Proc. of the Fifth Internat. Conf. on Computer Vision (20-23 June 1995) pp. 810-815.

[26] S. Kichenassamy, A. Kumar, P. Olver, A. Tannenbaum and A. Yezzi, Conformal curvature flows: From phase transitions to active vision, Archive Rational Mech. Anal. 134(3) (1996) 275-301.

[27] O.A. Ladyzhenskaya, V.A. Solonnikov and N.N. Ural'tseva, Linear and Quasilinear Equations of Parabolic Type (Amer. Math. Soc., Providence, RI, 1968).

[28] C. Le Guyader, Imagerie mathématique: Théory et applications, Thèse de doctorat, INSA Rouen (2004) en cours de rédaction.

[29] C. Le Guyader, D. Apprato and C. Gout, Using a level set approach for image segmentation under interpolation conditions, Numer. Algorithms (2003) submitted.

[30] R. Malladi and J.A. Sethian, Image processing via level curvature flow, Proc. National Acad. Sci. 92(15) (1995) 7046-7050.

[31] R. Malladi, J.A. Sethian and B.C. Vermuri, A fast level set based algorithm for topology independent shape modeling and recovery, in: Proc. of the 3rd ECCV, Stockholm, Sweden (1994) pp. 3-13.

[32] S. Osher and R. Fedkiw, Level Set Methods and Dynamic Implicit Surfaces (Springer, Berlin, 2003).

[33] S. Osher and J.A. Sethian, Fronts propagation with curvature dependent speed: Algorithms based on Hamilton-Jacobi formulations, J. Comput. Phys. 79 (1988) 12-49.

[34] J.A. Sethian, Level Set Methods and Fast Marching Methods: Evolving Interfaces in Computational Geometry, Fluid Mechanics, Computer Vision and Material Science (Cambridge Univ. Press, London, 1999).

[35] J.A. Sethian, Evolution, implementation and application of level set and fast marching methods for advancing fronts, J. Comput. Phys. 169(2) (2001) 503-555.

[36] J.A. Sethian, A review of recent numerical algorithms for surface s moving with curvature dependent flows, J. Differential Geometry 31 (1989) 131-161.

[37] L. Vese, A method to convexify functions via curve evolution, Commun. Partial Differential Equations 24(9/10) (1999) 1573.

[38] L.A. Vese, Study in the BV space of a denoising-deblurring variational problem, Appl. Math. Optim. 44(2) (2001) 131-162.

[39] L.A. Vese and T.F. Chan, A multiphase level set framework for image segmentation using the Mumford and Shah model, Internat. J. Computer Vision 50(3) (2002) 271-293.

[40] H.-K. Zhao, S. Osher, B. Merriman and M. Kang, Implicit and nonparametric shape reconstruction from unorganized data using a variational level set method, Computer Vision Image Understanding 80(3) (2000) 295-314.

[41] H.-K. Zhao, T. Chan, B. Merriman and S. Osher, A variational level set approach to multiphase motion, J. Comput. Phys. 127 (1996) 179-195. 